Western University

Scholarship@Western

$11-25-2019$

\title{
Accelerated 129Xe MRI morphometry of terminal airspace enlargement: Feasibility in volunteers and those with alpha-1 antitrypsin deficiency
}

Alexei Ouriadov

Fumin Guo

David G McCormack

Grace Parraga

Follow this and additional works at: https://ir.lib.uwo.ca/biophysicspub

Part of the Medical Biophysics Commons

Citation of this paper:

Ouriadov A, Guo F, McCormack DG, Parraga G. Accelerated 129Xe MRI morphometry of terminal airspace enlargement: Feasibility in volunteers and those with alpha-1 antitrypsin deficiency. Magn Reson Med. 2020;84: 416-426. https://doi.org/10.1002/mrm.28091 


\title{
Accelerated ${ }^{129}$ Xe MRI morphometry of terminal airspace enlargement: Feasibility in volunteers and those with alpha-1 antitrypsin deficiency
}

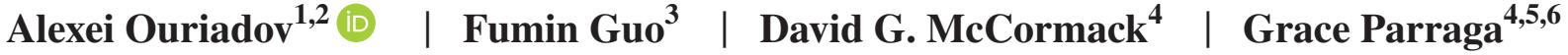 \\ ${ }^{1}$ Department of Physics and Astronomy, The University of Western Ontario, London, Canada \\ ${ }^{2}$ Lawson Health Research Institute, London, Canada \\ ${ }^{3}$ Sunnybrook Research Institute, University of Toronto, Toronto, Canada \\ ${ }^{4}$ Division of Respirology, Department of Medicine, The University of Western Ontario, London, Canada \\ ${ }^{5}$ Robarts Research Institute, The University of Western Ontario, London, Canada \\ ${ }^{6}$ Department of Medical Biophysics, The University of Western Ontario, London, Canada
}

\section{Correspondence}

Alexei Ouriadov, Department of Physics and Astronomy, PAB Room 270,

The University of Western Ontario,

1151 Richmond Street, London,

Ontario N6A 3K7, Canada.

Email: aouriado@uwo.ca

Funding information

Canadian Institutes of Health Research (CIHR), Grant/Award Number:

MOP 106437; Canadian Respiratory

Research Network; Natural Sciences and Engineering Research Council (Canada);

Alpha-1 Foundation (USA)
Purpose: Multi-b diffusion-weighted hyperpolarized inhaled-gas MRI provides imaging biomarkers of terminal airspace enlargement including ADC and mean linear intercept $\left(\mathrm{L}_{\mathrm{m}}\right)$, but clinical translation has been limited because image acquisition requires relatively long or multiple breath-holds that are not well-tolerated by patients. Therefore, we aimed to accelerate single breath-hold 3D multi-b diffusionweighted ${ }^{129} \mathrm{Xe} \mathrm{MRI}$, using $\mathrm{k}$-space undersampling in imaging direction using a different undersampling pattern for different $b$-values combined with the stretched exponential model to generate maps of ventilation, apparent transverse relaxation time constant $\left(\mathrm{T}_{2}^{*}\right), \mathrm{ADC}$, and $\mathrm{L}_{\mathrm{m}}$ values in a single, short breath-hold; accelerated and non-accelerated measurements were directly compared.

Methods: We evaluated multi-b $\left(0,12,20,30\right.$, and $\left.45.5 \mathrm{~s} / \mathrm{cm}^{2}\right)$ diffusion-weighted ${ }^{129} \mathrm{Xe} \mathrm{T}_{2}^{*} / \mathrm{ADC} /$ morphometry estimates using acceleration factor $(\mathrm{AF}=1$ and 7$)$ and multi-breath sampling in 3 volunteers (HV), and 6 participants with alpha-1 antitrypsin deficiency (AATD).

Results: For the HV subgroup, mean differences of 5\%, 2\%, and $8 \%$ were observed between fully sampled and undersampled k-space for ADC, $\mathrm{L}_{\mathrm{m}}$, and $\mathrm{T}_{2}^{*}$ values, respectively. For the AATD subgroup, mean differences were $9 \%, 6 \%$, and $12 \%$ between fully sampled and undersampled $\mathrm{k}$-space for $\mathrm{ADC}, \mathrm{L}_{\mathrm{m}}$ and $\mathrm{T}_{2}^{*}$ values, respectively. Although mean differences of $1 \%$ and $4.5 \%$ were observed between accelerated and multi-breath sampled $A D C$ and $L_{m}$ values, respectively, mean $A D C / L_{m}$ estimates were not significantly different from corresponding mean $A D C^{\mathrm{M}} / \mathrm{L}_{\mathrm{m}}{ }^{\mathrm{M}}$ or mean $\mathrm{ADC}^{\mathrm{A}} / \mathrm{L}_{\mathrm{m}}{ }^{\mathrm{A}}$ estimates (all $P>0.60,{ }^{\mathrm{A}}=$ undersampled and ${ }^{\mathrm{M}}=$ multi-breath sampled). 
Conclusions: Accelerated multi-b diffusion-weighted ${ }^{129} \mathrm{Xe}$ MRI is feasible at $\mathrm{AF}=7$ for generating pulmonary $\mathrm{ADC}$ and $\mathrm{L}_{\mathrm{m}}$ in AATD and normal lung.

K E Y W O R D S

acceleration, alpha-1 antitrypsin deficiency, compressed sensing, emphysema, hyperpolarized, lung, morphometry, xenon

\section{INTRODUCTION}

Hyperpolarized inhaled noble gas pulmonary $\mathrm{MRI}^{1,2}$ provides physiologically relevant biomarkers of obstructive lung disease including emphysema, bronchopulmonary dysplasia (BPD), congenital lobar emphysema, and alpha-1 antitrypsin deficiency (AATD). ${ }^{3-5}$ The ability to non-invasively characterize alveolar destruction without ionizing radiation makes hyperpolarized gas MRI a unique tool for frequent evaluation of lung disease progression and treatment outcomes in $\mathrm{BPD}^{6}$ and $\mathrm{AATD}{ }^{7}$ which commonly manifest in young or middle-age adulthood. A number of preliminary multi-b diffusion-weighted ${ }^{3} \mathrm{He}$ MRI studies in patients with $\mathrm{BPD}^{8}$ and $\mathrm{AATD}^{9}$ have provided strong evidence for the feasibility of lung morphometry measurements in patients with extensive disease. Moreover, ${ }^{129} \mathrm{Xe}$ ADCs were consistent with histologic measurements in chronic obstructive pulmonary disease (COPD) patients. ${ }^{10}$ Static ventilation mapping with relatively inexpensive, naturally abundant hyperpolarized ${ }^{129} \mathrm{Xe}$ is also feasible in patients. ${ }^{11}$ The physical properties of ${ }^{129} \mathrm{Xe}$ nuclei (e.g., low gyromagnetic ratio) require the use of rapid MRI acquisition strategies ${ }^{12-14}$ considering that the gradient strengths for clinical scanners is typically around $5 \mathrm{G} / \mathrm{cm}$. This is especially true in the case of multi-b diffusion-weighted MRI because currently, a full 3D data set cannot be acquired during the relatively short 10-16-s breathholds ${ }^{15}$ that patients can tolerate.

Recently, a stretched exponential model (SEM) ${ }^{16}$ combined with $\mathrm{k}$-space undersampling in the imaging direction, using a different undersampling pattern for different $b$-values and diffusion directions ${ }^{17}$ was used for the generation of ${ }^{3} \mathrm{He}$ static-ventilation, $\mathrm{T}_{2}^{*}$ and multiple b-value diffusionweighted MRI-based ADC and morphometry maps. ${ }^{18}$ The major advantage of this method is the possibility for decreased data acquisition times using acceleration factors (AF) between $7^{18}$ and $10 .{ }^{17}$ Because of the increasing scarcity and cost of ${ }^{3} \mathrm{He}$, most of the field is moving toward the use of ${ }^{129} \mathrm{Xe} \cdot{ }^{1,3,4}$ However, the low diffusivity of ${ }^{129} \mathrm{Xe}$ (or low xenon free diffusion coefficient $\left[\mathrm{D}_{0}\right]$ ) requires a longer diffusion-observation time $(\Delta)$ to sufficiently sensitize spins when using b-values up to $45 \mathrm{~s} / \mathrm{cm}^{2}$ as typical clinical gradient strengths are $5 \mathrm{G} / \mathrm{cm}$. In turn, a longer $\Delta$ leads to increased TE and consequently the TR, which ultimately increases the total scan time for ${ }^{129} \mathrm{Xe}$ MRI (>16 s, ethics-based breath-hold duration).
Therefore, they require that rapid acquisition strategies need to be developed to facilitate clinical uptake of ${ }^{129} \mathrm{Xe}$ diffusionweighted imaging. We hypothesize that the ${ }^{3} \mathrm{He}$ method ${ }^{17,18}$ can be modified to provide whole lung ${ }^{129} \mathrm{Xe}$ MRI-based emphysema biomarkers including static-ventilation, $\mathrm{T}_{2}^{*}$, ${ }^{19-21}$ ADC and morphometry maps. Therefore, in this proofof-concept evaluation, our objective was to demonstrate the feasibility of this approach in a small group of patients with lung disease and in volunteers by conducting a back-to-back comparison of the single breath hold undersampled k-space (UKS) data set with the single breath-hold fully sampled k-space (FKS) data set and with the multiple-breath k-space (MKS) data set. To our knowledge, this is the first demonstration in patients of simultaneous ${ }^{129} \mathrm{Xe}$ lung morphometry, gas density distribution, and $\mathrm{T}_{2}^{*}$ mapping, resulting in physiologically relevant measurements, accomplished using a stretched exponential method with image acceleration.

\section{$2 \mid$ METHODS}

\section{1 | Study participants}

Three never-smokers (young healthy volunteers [HV]) and 6 AATD patients provided written informed consent to the research ethics board, compliant with the Health Insurance Portability and Accountability Act (HIPAA, USA). Subject demographics are summarized in Table 1 and in Supporting Information Table S1. Each AATD subject had 2 hyperpolarized gas MRI scans within $4 \mathrm{y}$ : a ${ }^{3} \mathrm{He}$ MRI scan in $2014^{9}$ and ${ }^{129}$ Xe MRI scan in 2018 (this study). The HV subjects did not have any ${ }^{3} \mathrm{He}$ MRI scans in 2014 or 2018, they had only a ${ }^{129}$ Xe MRI scan in 2018 (this study).

\section{2 | Pulmonary function tests}

Spirometry, plethysmography, and the diffusing capacity of the lung for carbon monoxide $\left(\mathrm{DL}_{\mathrm{CO}}\right)$ were performed according to American Thoracic Society (ATS) guidelines ${ }^{22}$ using a plethysmograph and attached gas analyzer (MedGraphics, St. Paul, USA). Table 1 and Supporting Information Table S1 summarize spirometry, plethysmography, and $\mathrm{DL}_{\mathrm{CO}}$ for all subjects. 
T A B L E 1 Pulmonary function measurements, demographic, and ${ }^{129}$ Xe MRI parenchyma measurements

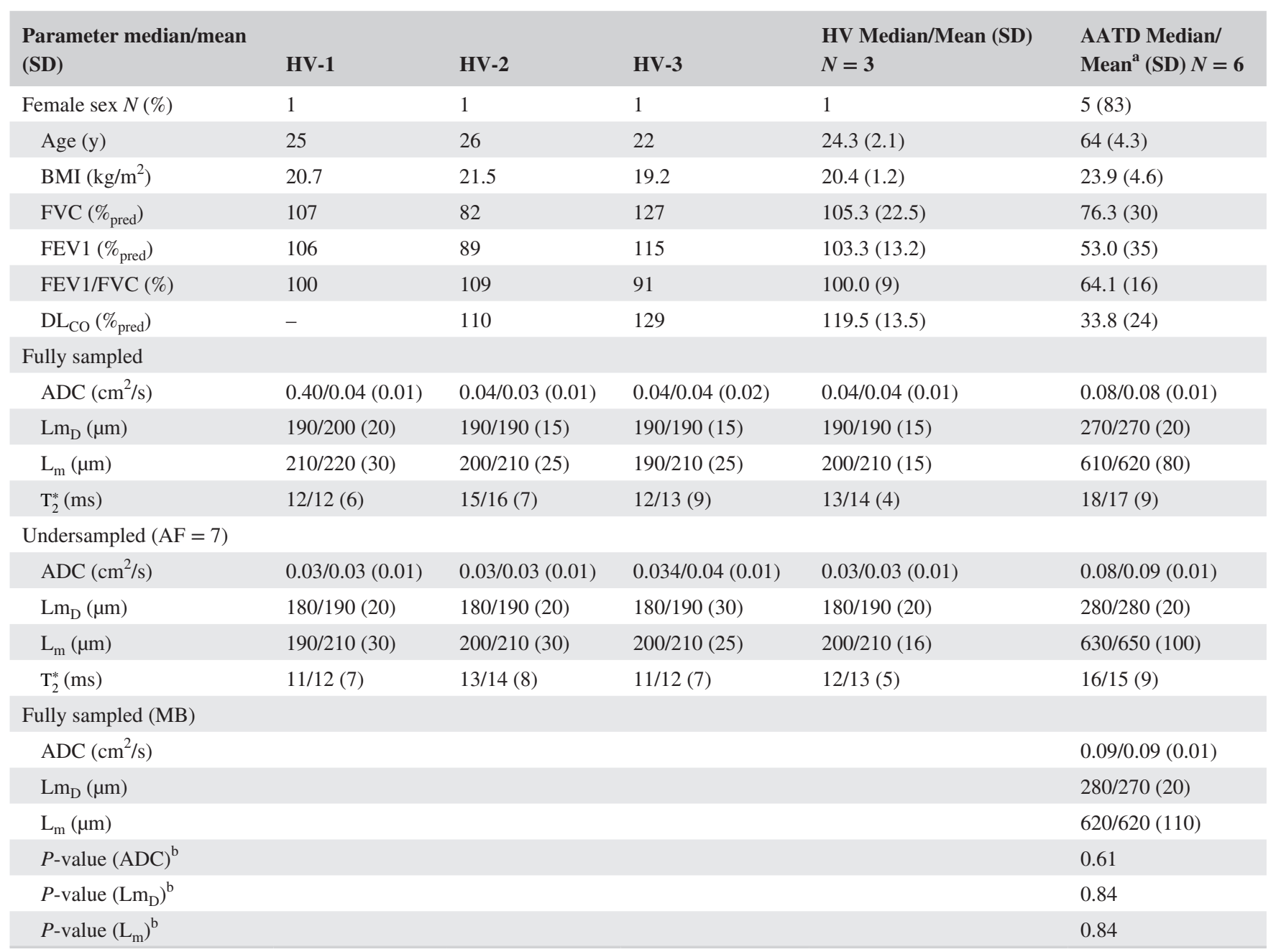

Abbreviations: AATD, alpha-one antitrypsin deficiency; $\mathrm{AF}$, acceleration factor; $\mathrm{BMI}$, body mass index; $\mathrm{DL}_{\mathrm{CO}}$, diffusing capacity of carbon monoxide; $\mathrm{FEV} \mathrm{V}_{1}$, forced expiratory volume in $1 \mathrm{~s} ; \mathrm{FVC}$, forced-vital-capacity; HV, young healthy volunteer; $\mathrm{L}_{\mathrm{m}}$, mean linear intercept estimate; $\mathrm{Lm}_{\mathrm{D}}$, mean diffusion length; MB, multi-breath data acquisition; $\%_{\text {pred}}$, percent-predicted; RV, residual-volume; $\mathrm{T}_{2}^{*}$, apparent transverse relaxation time constant.

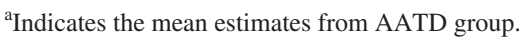

${ }^{\mathrm{b}}$ MANOVA.

\section{$2.3 \quad{ }^{129}$ Xe MRI}

${ }^{129} \mathrm{Xe}$ MRI was performed at 3.0T (MR750, GEHC, Waukesha, USA) using whole-body gradients $(5 \mathrm{G} / \mathrm{cm}$ maximum) and a commercial, xenon quadrature flex human RF coil ${ }^{12}$ (Clinical MR Solutions, Brookfield, USA). The diffusion-sensitization gradient pulse ramp up/down time = $500 \mu \mathrm{s}$, constant time $=2 \mathrm{~ms}, \Delta_{\mathrm{Xe}}=5.2 \mathrm{~ms}$, providing $5 \mathrm{~b}$-values $0,12.0,20.0,30.0$, and $45.5 \mathrm{~s} / \mathrm{cm}^{2}$. For fully sampled acquisitions (single breath-hold), a multi-slice centric 2D fast gradient recalled echo (FGRE) diffusion-weighted sequence (Figure 1A) was used with 5 b-values, sequentially acquired for each k-space line and slice. Additional sequence parameters include $230-\mathrm{mm}$ central coronal slices, TE = $10 \mathrm{~ms}, \mathrm{TR}=13 \mathrm{~ms}$, reconstructed matrix size $=128 \times 128$, and FOV $=40 \times 40 \mathrm{~cm}^{2}$, constant-flip-angle $=4^{\circ}$, and 14-s single breath-hold. For an accelerated acquisition $(\mathrm{AF}=7)$, multi-slice centric 2D FGRE diffusion-weighted sequence (Figure 1A) was acquired with 5 b-values, sequentially acquired for each $\mathrm{k}$-space line and slice, using undersampling in the imaging direction with different undersampling patterns for different b-value as shown in Figure 1C (bottom panel). Additional sequence parameters were similar to the fully sampled case except the matrix size was $128 \times 20$. In both accelerated and unaccelerated cases an extra sequential image (for each slice) with no diffusion-weighting $(b=0)$ and significantly reduced TE $(2 \mathrm{~ms})$ was used to generate a short-TE static-ventilation (SV) image (Figure 1B). A $7.4^{\circ}$ constant flip angle (120 [20 per b-value] RF pulses-per-slice, $730 \mathrm{~mm}$ coronal slices) was used for $\mathrm{AF}=7$ (all participants, 12-s single breath-hold). In both cases, a diffusionsensitizing scheme initiating at the maximum b-value was used to ensure that maximum MR signal was acquired for diffusion-weighted images at greater b-values as previously 


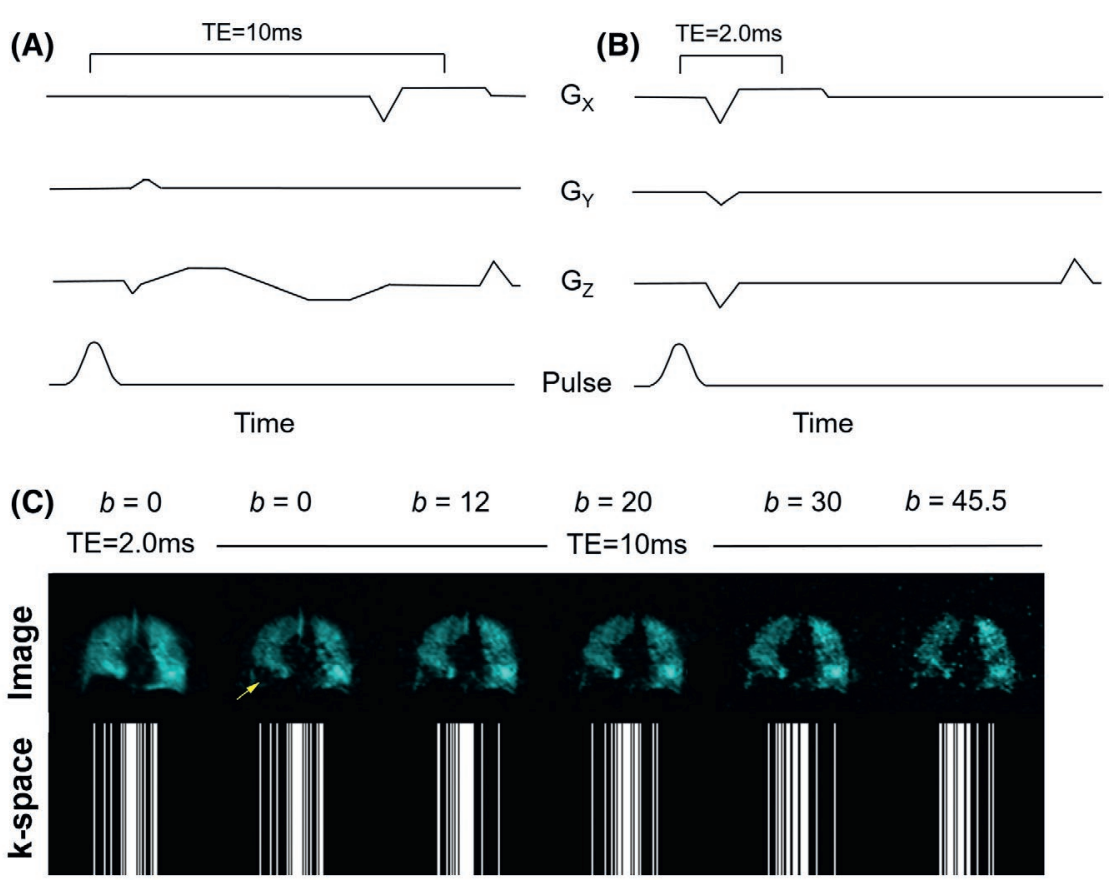

F I G U RE $1 \quad{ }^{129}$ Xe MRI pulse sequence schematic, sparsity pattern for AF = 7. (A) Diffusion-weighted, multi-slice $2 \mathrm{D}$ fast-gradientrecall-echo (FGRE) pulse sequence with diffusion-sensitizing along z-direction. $\Delta=5.2 \mathrm{~ms}$, TE $=10 \mathrm{~ms}$. Five b-values, starting at the maximum b-value $\left(45.5 \mathrm{~s} / \mathrm{cm}^{2}\right)$ were sequentially acquired for the same line of $\mathrm{k}$-space for each slice. (B) An extra sequential image with no diffusionweighting $(\mathrm{b}=0)$ and significantly reduced TE $(2 \mathrm{~ms})$ used to generate a short TE static-ventilation-image and $\mathrm{T}_{2}^{*}$ map by using a long TE static ventilation image $(b=0)$ from $(A)$. (C) A short TE static ventilation image, a long TE static ventilation image, and 4 diffusion-weighted images (top panel) shown from left to right. The images were obtained from a young healthy volunteer and they are individually scaled. The SNR value varies from 60 (short TE static ventilation image) to 12 (maximum b-value image). The yellow arrow shows a region with the significant $\mathrm{T}_{2}^{*}$-based signal decay; k-space undersampling scheme, ensuring a variety of sparsity patterns for each b-value (AF $=7$ bottom panel) used in diffusion direction

described.$^{9}$ A $5.0^{\circ}$ constant flip angle was used in all multibreath measurements.

For AATD participants, fully sampled acquisitions were also used using 3 breath-holds, a multi-slice interleaved (2 interleaves), centric 2DFGRE diffusion-weighted sequence to acquire $730-\mathrm{mm}$ coronal slices (additional sequence parameters were similar to fully sampled, 10 -s single breathhold) using $4 \mathrm{~b}$-values ( 0 and $12 \mathrm{~s} / \mathrm{cm}^{2} ; 0$ and $20 \mathrm{~s} / \mathrm{cm}^{2}$; and 0 and $30 \mathrm{~s} / \mathrm{cm}^{2}$ ).

In addition to the single-breath imaging under accelerated and unaccelerated cases, the multi-breath approach required extra xenon doses, much longer MRI-idle times and personnel involvement, so only the AATD patients were scanned with this method. ${ }^{18}$

All participants were coached to perform the breathhold maneuvers to minimize potential for mis-registration and differences in the levels of inspiration between the scans. ${ }^{15,23}$ Hyperpolarized ${ }^{129} \mathrm{Xe}$ gas $(86 \%$ enriched, measured polarization $\sim 40 \%$ ) was provided by a commercial xenon polarizer system (Model 9820, Polarean, Durham, USA). ${ }^{24}$ Subjects were positioned supine in the scanner for ${ }^{129}$ Xe MRI. For all participants, a single $1 \mathrm{~L}$ plastic bag (Tedlar, Jensen Inert Products, Coral Springs, USA) containing a $50 / 50$ hyperpolarized ${ }^{129} \mathrm{Xe} /{ }^{4} \mathrm{He}$ gas mixture was inhaled 5 times (for AATD patients) or 2 times (for HV) from functional residual capacity (e.g., heldbreath volume of functional residual capacity plus $1 \mathrm{~L}) .^{15,23}$

The scanner was occupied for up to $2 \mathrm{~h}$ (proton scans, xenon scans, and MRI-idle time) depending on the number of xenon doses. All participants (HV and ADDT) were only in the supine position for up to $20 \mathrm{~min}$ at a time during scanning and were upright between ${ }^{129} \mathrm{Xe}$ scans. Participants were encouraged to sit up and walk around between acquisitions and polarized xenon collections ( $20 \mathrm{~min}$ ) because previous studies confirmed that lung imaging results might not be a physiologically relevant after spending relatively long time in supine position. ${ }^{25,26}$ Therefore, we had to remove the patient from the scanner after each xenon dose or scan and relocalize (proton scan) the patient for the next xenon scan. Such approach likely led to a slight slice mismatch and potentially slight lung volume mismatch between the different xenon scans.

\section{4 | Data analysis}

The experimental signal at particular b-value values can be fit with the SEM, which was derived for multi-b diffusionweighted ${ }^{3} \mathrm{He}$ MRI for lung imaging ${ }^{9,16-18}$ as follows 


$$
\mathrm{S}(\mathrm{b}) / \mathrm{S}_{0}=\exp \left(-\mathrm{D}^{\prime} \cdot \overline{\mathrm{b}}\right)^{\alpha}
$$

where $\mathrm{D}^{\prime}$ is the apparent diffusivity, $\alpha$ is the heterogeneity index $(0<\alpha \leq 1.0)$ and $\mathrm{S}_{0}$ is the MR signal-intensity in the absence of diffusion-sensitizing gradients. $\mathrm{D}^{\prime}$ and $\alpha$ estimates can be used to calculate specific acinar duct mean diffusion length estimates $^{27}\left(\mathrm{Lm}_{\mathrm{D}}=\sqrt{2 \Delta \mathrm{D}}\right.$, where $\Delta$ is the diffusion time and $\mathrm{D}$ is the most probable diffusion coefficient) using the probability density function or diffusion propagator., ${ }^{9,27}$ For the multi-b diffusion-weighted ${ }^{3} \mathrm{He}$ MRI case, $\mathrm{L}_{\mathrm{m}}$ is proportional to $\mathrm{Lm}_{\mathrm{D}}{ }^{9,18}$

$$
\mathrm{L}_{\mathrm{m}}=-562 \mu \mathrm{m}+4.3 \cdot \mathrm{Lm}_{\mathrm{D}} \text {. }
$$

Equation 2 was obtained for the ${ }^{3} \mathrm{He}$ case and a specific $\Delta$. Generally, the mean airway length depends on both $\Delta$ and diffusivity, and Equation 2 cannot be directly used for calculation of ${ }^{129}$ Xe MRI $L_{m}$ estimates. Note that $L_{m}$ is a time-gas independent parameter. To extend Equation 2 to other $\Delta$ and to other gases, particularly to ${ }^{129} \mathrm{Xe}$ gas the following empirical relation was proposed ${ }^{18,29}$

$$
\mathrm{L}_{\mathrm{m}}=-562 \mu \mathrm{m}+4.3 \cdot \mathrm{Lm}_{\mathrm{D}} \cdot \sqrt{\frac{2 \mathrm{D}_{0}^{\mathrm{He}} \Delta_{\mathrm{He}}}{2 \mathrm{D}_{0}^{\mathrm{Xe}} \Delta_{\mathrm{Xe}}}},
$$

where $\mathrm{D}_{0}^{\mathrm{He}}$ is the free diffusion coefficient of ${ }^{3} \mathrm{He}\left(0.88 \mathrm{~cm}^{2} / \mathrm{s}\right)$, $\Delta_{\mathrm{He}}=1.46 \mathrm{~ms},{ }^{9,18} \mathrm{D}_{0}^{\mathrm{Xe}}$ is the free diffusion coefficient of ${ }^{129} \mathrm{Xe}\left(0.21 \mathrm{~cm}^{2} \mathrm{~s}^{-1}\right)^{3}$ in the human lung $\left(1 \mathrm{~L}\right.$ of ${ }^{129} \mathrm{Xe} /{ }^{4} \mathrm{He}$ diluted by air) and $\Delta_{\mathrm{Xe}}$ is the diffusion time specific to the diffusion-weighted ${ }^{129} \mathrm{Xe}$ MRI measurements.

${ }^{129} \mathrm{Xe}$ undersampled data $(\mathrm{AF}=7)$ were reconstructed ${ }^{17}$ using the regularization parameters previously determined. ${ }^{30}$ A single slice image ( $5 \mathrm{~b}$-values and short TE) reconstruction was completed within $\sim 2-3$ min using MATLAB $2018 \mathrm{~b}$ (MathWorks, Natick, USA) on a standard PC workstation with a $3.0 \mathrm{GHz}$ CPU using a previously developed approach. ${ }^{17}$ Reconstructed data and fully sampled data were fitted with the SEM model to generate $\mathrm{D}^{\prime}, \alpha$, and $\mathrm{Lm}_{\mathrm{D}}$ maps on a voxelby-voxel basis. ${ }^{17,18}$ For AATD patients the $\mathrm{L}_{\mathrm{m}}$ maps were generated using Equation 3, for young healthy volunteers $\mathrm{L}_{\mathrm{m}}$ maps were generated using the cylindrical model $(\mathrm{CM})^{31-33}$ as empirical Equations 2 and 3 were obtained for older subjects. ${ }^{9}$ The ADC maps were generated for $2 \mathrm{~b}$-values $\left(0\right.$ and $\left.12 \mathrm{~s} / \mathrm{cm}^{2}\right)$ on a voxel-by-voxel basis. The choice of the particular b-values for the xenon ADC calculations was previously discussed. ${ }^{5} \mathrm{~T}_{2}^{*}$ maps were generated using short-TE (2 ms) static-ventilationimage $(b=0)$ and long-TE $(10 \mathrm{~ms})$ static-ventilation-image $(b=0)$ (Figure 1C, top panel) on a voxel-by-voxel basis for both accelerated and fully sampled cases. The large airways were excluded from the $\mathrm{T}_{2}^{*} / \mathrm{ADC} /$ morphometry analyses.

\section{5 | Statistical analysis}

Multivariate analysis of variance (MANOVA) and independent t-tests were performed using SPSS Statistics, V22.0
F IGURE 2 Representative

${ }^{129}$ Xe MRI maps obtained for 2 healthy volunteers with and without acceleration. $\mathrm{T}_{2}^{*}$, apparent transverse relaxation time constant; ADC, apparent diffusion coefficient; $\mathrm{Lm}_{\mathrm{D}}$, mean diffusion length; $\mathrm{L}_{\mathrm{m}}$, mean linear intercept estimate; $\mathrm{HV}$, young healthy volunteer; ${ }^{\mathrm{A}}$, indicates undersampling with the acceleration factor of 7. Note that some differences in the lower right lobe for $\mathrm{HV}-2$ compared to $\mathrm{HV}^{\mathrm{A}}-2$ are likely because of the slice and lung volume mismatch between accelerated and fullsampled data sets

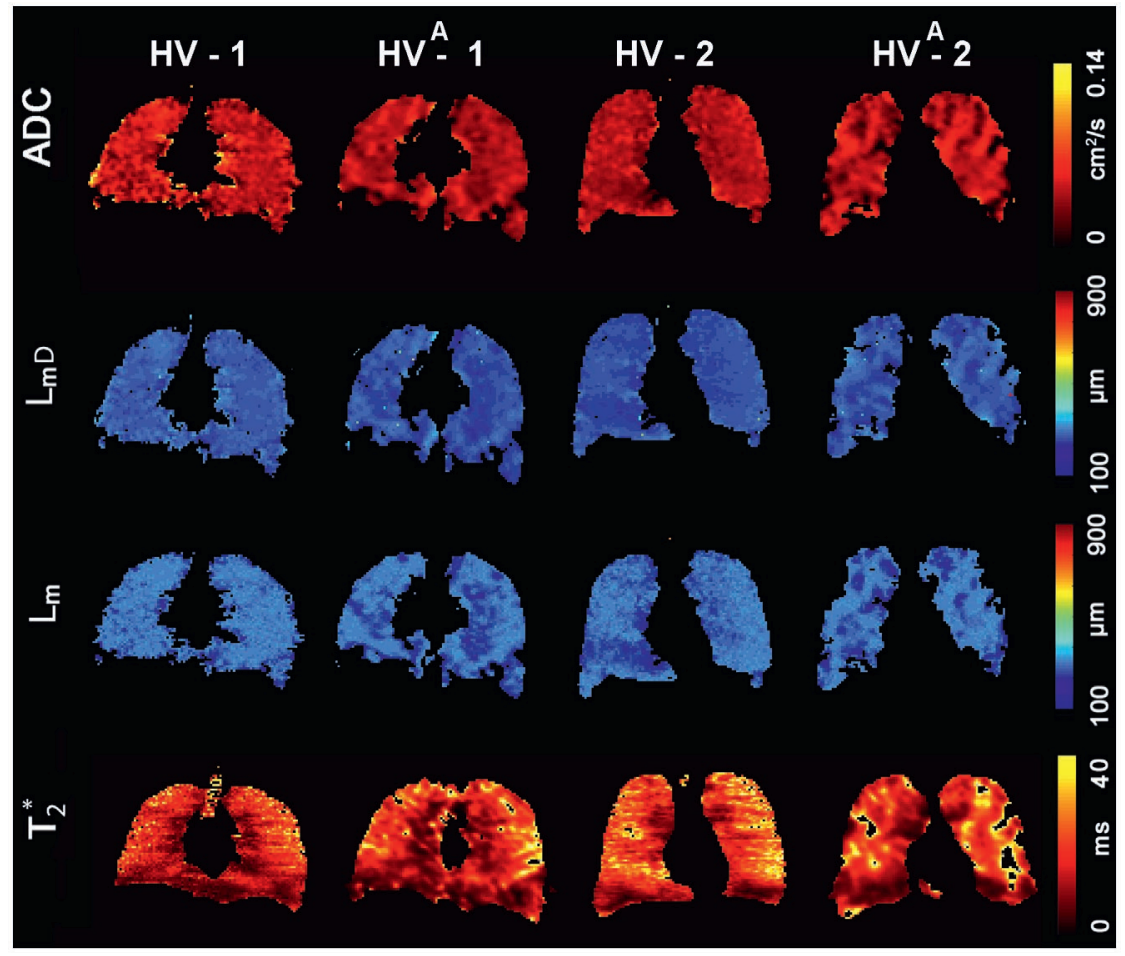


(SPSS Inc., Chicago, USA) and results were considered statistically significant when the probability of making a type I error was $<5 \%(P<.05)$.

\section{3 | RESULTS}

Figure 1C (bottom panel) shows a representative center slice reconstructed from undersampling data for a single AATD patient. Differences in signal intensity were visibly obvious because of $\mathrm{T}_{2}^{*}$ decay and diffusion attenuation. The minimum SNR value for all acquired data sets varied from 60 (short TE static ventilation image) to 10 (maximum b-value image).
The yellow arrow indicated the lung region with ventilation defect-like signal void observed for the long-TE image, but not observed for the short-TE image.

Figure 2 ( ${ }^{\mathrm{A}}$ indicates accelerated sampling) shows representative center slice ADC, $\mathrm{Lm}_{\mathrm{D}}, \mathrm{L}_{\mathrm{m}}$, and $\mathrm{T}_{2}^{*}$ maps for both unaccelerated and accelerated cases for 2 volunteers. For the HV subgroup, mean differences of $5 \%, 1 \%, 2 \%$, and $8 \%$ were observed between fully sampled and undersampled (AF $=1$ and $\mathrm{AF}=7$ ) $\mathrm{k}$-space for the $\mathrm{ADC}, \mathrm{Lm}_{\mathrm{D}}, \mathrm{L}_{\mathrm{m}}$, and $\mathrm{T}_{2}^{*}$ values, respectively.

Figure 3 ( ${ }^{\mathrm{A}_{\text {indicates }}}$ multi-breath fully sampled data) shows a representative center slice $\mathrm{ADC}, \mathrm{Lm}_{\mathrm{D}}$, and $\mathrm{L}_{\mathrm{m}}$ map for the unaccelerated and accelerated cases using a single
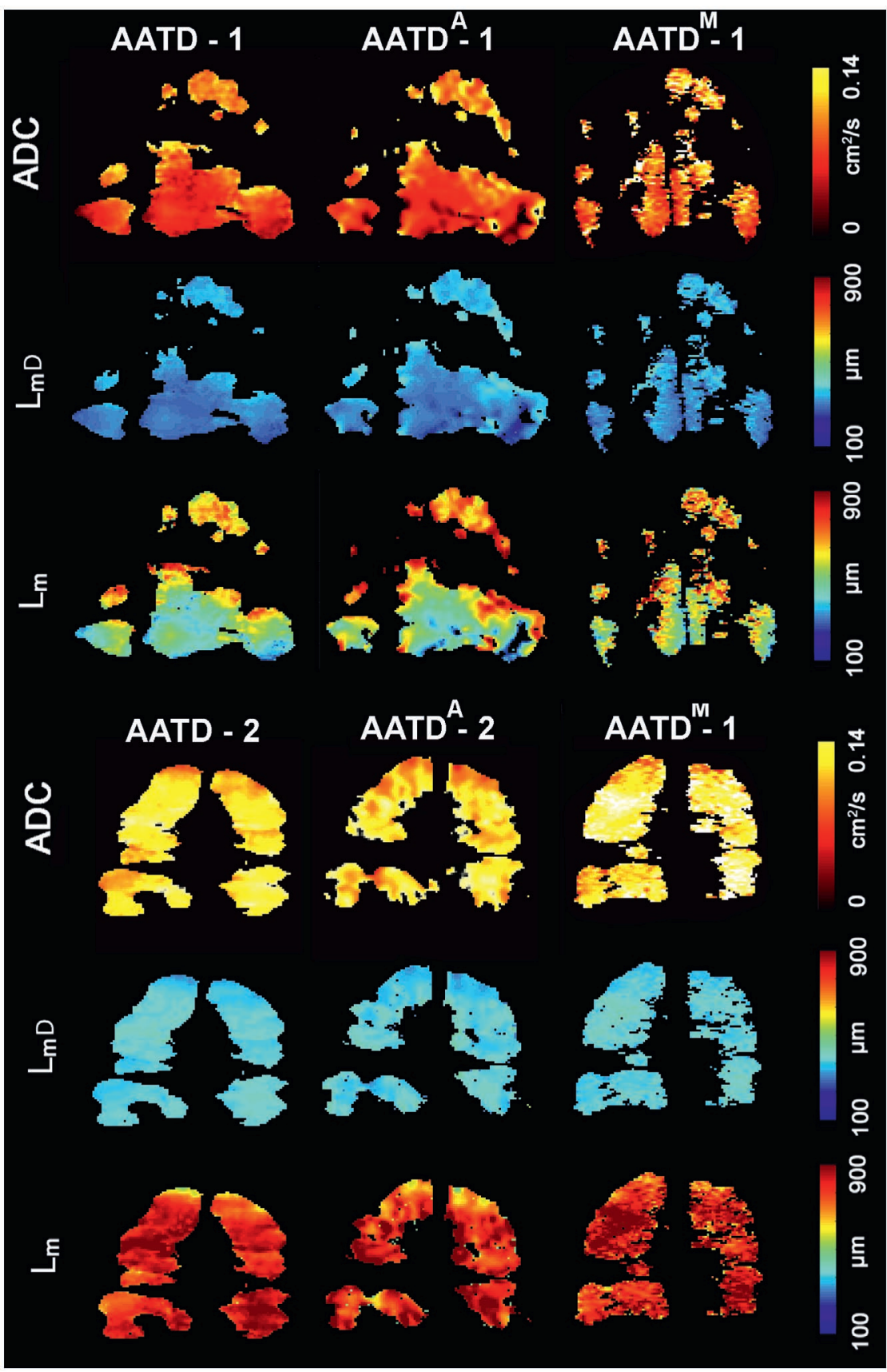

F IG URE 3 Representative ${ }^{129} \mathrm{Xe}$ MRI maps obtained for 2 AATD with and without acceleration at a single breath-hold and multiple breaths (no acceleration). ADC, apparent diffusion coefficient; $\mathrm{Lm}_{\mathrm{D}}$, mean diffusion length; $\mathrm{L}_{\mathrm{m}}$, mean linear intercept estimate; AATD, alpha-one antitrypsin deficiency; AATD-2, ex-smoker AATD; ${ }^{\mathrm{A}}$, indicates undersampling with the acceleration factor of $7 ;^{\mathrm{M}}$, indicates multiple breath-holds, no acceleration. Note that AATD-1 and AATD ${ }^{\mathrm{A}}-1$ maps (single breath-hold acquisition) are clearly different from the AATD ${ }^{\mathrm{M}}$-1 map (multiple breath-hold acquisition) in the ventilation likely because of the unavoidable slice and lung volume mismatch, the actual parameter maps are fairly similar 


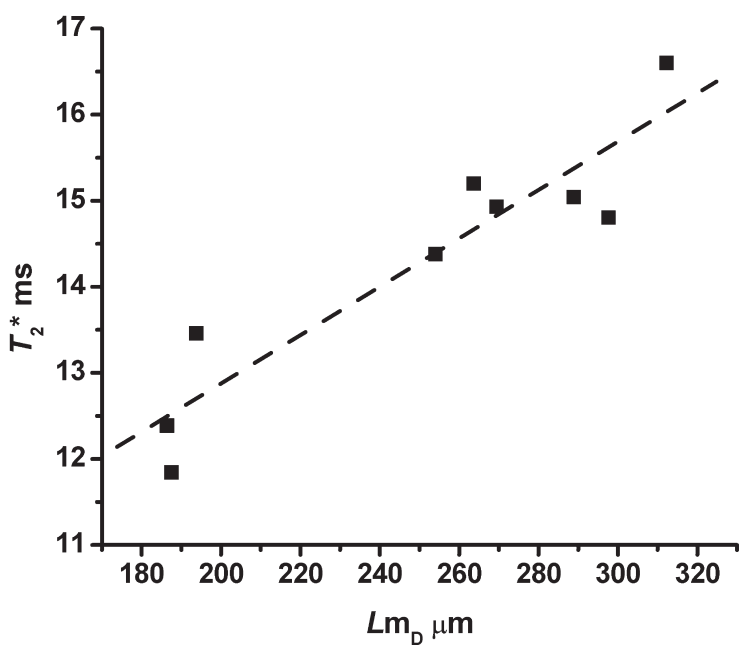

F I G U R E 4 Relationship for $\mathrm{Lm}_{\mathrm{D}}=$ mean diffusion length with $\mathrm{T}_{2}^{*}$ for all study participants $(\mathrm{R}=0.93 ; \mathrm{y}=0.03 \mathrm{x}+7.3 \mathrm{~ms}$; $P<.001)$. The plot suggests that alveolar destruction improves $\mathrm{B}_{0}$ field homogeneity, so relatively slow moving xenon molecules do not experience a large scale $\mathrm{B}_{0}$ field inhomogeneity at $\mathrm{TE}=10 \mathrm{~ms}$ and as a result it makes the signal life-time longer

breath-hold, and also the unaccelerated multi-breath approach maps for 2 AATD subjects. For the AATD subgroup, mean differences of $4 \%, 2 \%$, and $4 \%$ were observed between fully sampled and undersampled $(\mathrm{AF}=1$ and $\mathrm{AF}=7) \mathrm{k}$-space for the $\mathrm{ADC}, \mathrm{Lm}_{\mathrm{D}}$, and $\mathrm{L}_{\mathrm{m}}$ values, respectively. In addition, mean differences of $1 \%, 3 \%$, and $5 \%$ were observed between accelerated and multi-breath samplings for the $\mathrm{ADC}, \mathrm{Lm}_{\mathrm{D}}$, and $\mathrm{L}_{\mathrm{m}}$ values, respectively. Mean $\mathrm{ADC}, \mathrm{Lm}_{\mathrm{D}}$, and $\mathrm{L}_{\mathrm{m}}$ estimates were not significantly different from corresponding mean $\mathrm{ADC}^{\mathrm{M}}, \mathrm{Lm}_{\mathrm{D}}{ }^{\mathrm{M}}$, and $\mathrm{L}_{\mathrm{m}}{ }^{\mathrm{M}}$ and mean $\mathrm{ADC}^{\mathrm{A}}, \mathrm{Lm}_{\mathrm{D}}{ }^{\mathrm{A}}$, and $\mathrm{L}_{\mathrm{m}}{ }^{\mathrm{A}}$ estimates $(P>0.60$; for all). Supporting Information Figure $\mathrm{S} 1 \mathrm{~A}$ and $\mathrm{B}$ show the $\mathrm{ADC}^{\mathrm{A}}$ versus $\mathrm{ADC}(\mathrm{R}=0.67, P<.0001)$ and $\mathrm{Lm}_{\mathrm{D}}{ }^{\mathrm{A}}$ versus $\mathrm{Lm}_{\mathrm{D}}(\mathrm{R}=0.65, P<.0001)$ relations obtained for all AATD subjects and all pixels. Supporting Information Figure S2A and $\mathrm{B}$ show the $\mathrm{ADC}$ and $\mathrm{Lm}_{\mathrm{D}}$ statistical plots obtained for AATD-1 subject (Figure 3). Supporting Information Figure $\mathrm{S} 3 \mathrm{~A}$ and $\mathrm{B}$ show the $\mathrm{ADC}$ and $\mathrm{Lm}_{\mathrm{D}}$ statistical plots obtained for AATD-2 subject (Figure 3). Table 1 summarizes mean, median, and $\mathrm{SD} A D C, \mathrm{Lm}_{\mathrm{D}}, \mathrm{L}_{\mathrm{m}}$, and $\mathrm{T}_{2}^{*}$ values along with the pulmonary function test results obtained for young healthy volunteers and AATD patients. Supporting Information Table $\mathrm{S} 1$ shows mean, median, and SD estimates $\mathrm{ADC}, \mathrm{Lm}_{\mathrm{D}}, \mathrm{L}_{\mathrm{m}}$, and $\mathrm{T}_{2}^{*}$ as well as $\mathrm{D}^{\prime}$ and $\alpha$ for each AATD subject.

Lung heterogeneity for all study subjects can be estimated using the SD obtained from the ADC and morphometry results (Table 1; Supporting Information Table S1). In addition, Supporting Information Figure S4A and B show the ADC histograms (fully sampled ADC data) obtained for $2 \mathrm{HV}$ and 2 AATD subjects presented in Figures 2 and 3. The histograms obtained for HV showing an almost normal distribution of the ADC values, in contrast with the histograms obtained for the AATD subjects showing broad and rather the multi-peak distributions shifted to the high end of the ADC values.

Figure 4 shows an empirical relationship between mean $\mathrm{Lm}_{\mathrm{D}}$ and $\mathrm{T}_{2}^{*}$ estimates obtained for all study participants using undersampled ${ }^{129} \mathrm{Xe}$ MRI morphometry $(\mathrm{R}=0.93$; $\mathrm{y}=0.03 \mathrm{x}+7.3 \mathrm{~ms} ; P<.001)$. The plot clearly shows 2 groups of subjects where smaller $\mathrm{T}_{2}^{*}$ values $(<14 \mathrm{~ms})$ correspond to healthy lung parenchyma $\left(\operatorname{Lm}_{\mathrm{D}}<190 \mu \mathrm{m}\right)$ and longer $\mathrm{T}_{2}^{*}$ values $(>14 \mathrm{~ms}$ ) correspond to emphysematous lung tissue $\left(\mathrm{Lm}_{\mathrm{D}}>250 \mu \mathrm{m}\right)$.

\section{4 | DISCUSSION}

In this proof-of-concept study, we investigated the feasibility of accelerated SEM-based ${ }^{129}$ Xe MRI morphometry using $\mathrm{AF}=7$, allowing generation of an extended number of biomarkers including static-ventilation, $\mathrm{T}_{2}^{*}$, ADC, and morphometry. We also studied the influence of acceleration factors of multi-b diffusion-weighted ${ }^{129} \mathrm{Xe}$ MRI lung on $\mathrm{T}_{2}^{*}$, ADC, and SEM-based morphometry estimates using 3 different k-space sampling approaches in 9 participants including young healthy never-smokers and AATD patients. We made a number of observations including (1) accelerated SEM-based diffusion-weighted ${ }^{129} \mathrm{Xe}$ MRI was feasible and enabled collection of whole lung 2D multi-slice data sets in a single breath-hold, and (2) the extended ${ }^{129} \mathrm{Xe}$ stretchedexponential method provided estimates of the mean linear intercept in volunteers and patients with emphysema that were within known physiologically expected ranges.

Although AATD patients were previously studied using ${ }^{3} \mathrm{He}$ ADC/morphometry, ${ }^{9,34-37}$ to our knowledge, this is the first demonstration of the SEM-based lung morphometry obtained with $7 \times$ acceleration of multi-b diffusion-weighted ${ }^{129} \mathrm{Xe}$ MRI across a spectrum of emphysema severity.

It is also the first prospective evidence ${ }^{18}$ that an extended ${ }^{129} \mathrm{Xe}$ stretched exponential method, based on the cylindrical model, ${ }^{9,33}$ might be used for calculation of the $\mathrm{L}_{\mathrm{m}}$ estimations (as part of the SEM-based image reconstruction) along with CM. ${ }^{15,38}$ This is important in light of the clinical translation of ${ }^{129}$ Xe MRI morphometry and developing new tools for observation and characterization of emphysema.

First, we observed that SEM-based CS k-space acquisition and reconstructions resulted in ADC and morphometry estimates similar to those obtained for single and multiple breath-hold (AATD case) fully sampled cases and using Fast Fourier transform reconstruction. For AATD subgroups, FKS, MKS, and UKS estimates of $\mathrm{ADC}, \mathrm{Lm}_{\mathrm{D}}$, and $\mathrm{L}_{\mathrm{m}}$ were not significantly different $(P>0.61$, all cases, Table 1$)$. The difference in $\mathrm{ADC}$ and $\mathrm{Lm}_{\mathrm{D}}$ estimates obtained for FKS and UKS (HV: $5 \% / 1 \%, \mathrm{ADC} / \mathrm{Lm}_{\mathrm{D}}$ ) was similar to that observed 
with accelerated $(\mathrm{AF}=3){ }^{3} \mathrm{He}$ multi-b diffusion-weighted MRI in healthy subjects. ${ }^{16}$ For the AATD subgroup, the differences in ADC and $\mathrm{Lm}_{\mathrm{D}}$ estimates obtained for FKS and UKS were $3.8 \%$ and $2.0 \%$ and $1 \%$ and $3 \%$ for FKS and MKS, respectively.

Second, we demonstrated that the ${ }^{129} \mathrm{Xe}$ stretchedexponential method provided $\mathrm{L}_{\mathrm{m}}$ estimates that were within the expected physiologically range. ${ }^{9}$ The similarity between the SEM-based $\mathrm{L}_{\mathrm{m}}$ values and the $\mathrm{L}_{\mathrm{m}}$ values calculated for all of the study subjects using histologically validated CM (data not shown here) likely confirms this.

As previously shown, ${ }^{3} \mathrm{He}$ SEM provides morphometry estimates (Equation 2) similar to histology-based estimates. ${ }^{9}$ The empirical Equation 3 was used to calculate ${ }^{129}$ Xe MRI base $\mathrm{L}_{\mathrm{m}}$ estimates (Figure 3) instead of the time- and gasdependent Equation 2, which was validated with ${ }^{3} \mathrm{He}$ MRI for a large group of patients with a wide range of emphysema severity. ${ }^{9}$ The mean $\mathrm{L}_{\mathrm{m}}$ estimates obtained for 6 AATD subjects with ${ }^{3} \mathrm{He}$ MRI $\left.(2014)\right)^{9}$ and ${ }^{129} \mathrm{Xe}$ MRI (this study FKS, UKS, and MKS) were similar $(620 \pm 190 \mu \mathrm{m}$ vs. $620 \pm$ $80 \mu \mathrm{m} / 650 \pm 100 \mu \mathrm{m} / 620 \pm 110 \mu \mathrm{m}$, for ${ }^{3} \mathrm{He}$ [Table S1] ${ }^{9}$ and ${ }^{129} \mathrm{Xe}$ [Table 1; Supporting Information Table S1], respectively) taking into account a slight slice mismatch. There was no difference between $\mathrm{Lm}_{\mathrm{D}}^{\mathrm{He}}(2014)^{9}$ and $\mathrm{Lm}_{\mathrm{D}}^{\mathrm{xe}}$ estimates $(270 \pm 50 \mu \mathrm{m}$ vs. $270 \pm 20 \mu \mathrm{m} / 280 \pm 20 \mu \mathrm{m} / 270 \pm 20 \mu \mathrm{m}$, for ${ }^{3} \mathrm{He}$ [Table S1] ${ }^{9}$ and ${ }^{129} \mathrm{Xe}$ [Table 1; Supporting Information Table S1], respectively) generated for these AATD subjects. This was more likely because of the $\Delta / \mathrm{D}_{0}$ dependence of the specific airway mean diffusion length scale and the choice of diffusion parameters: $\mathrm{D}_{0}^{\mathrm{He}}=0.88$ $\mathrm{cm}^{2} / \mathrm{s}, \Delta_{\mathrm{He}}=1.46 \mathrm{~ms}^{9}$ and $\mathrm{D}_{0}^{\mathrm{Xe}}=0.21 \mathrm{~cm}^{2} \mathrm{~s}^{-1},{ }^{3} \Delta_{\mathrm{Xe}}=5.2$ $\mathrm{ms}$, therefore, $\sqrt{2 \mathrm{D}_{0}^{\mathrm{He}} \Delta_{\mathrm{He}}} \approx \sqrt{2 \mathrm{D}_{0}^{\mathrm{Xe}} \Delta_{\mathrm{Xe}}} \approx 500 \mu \mathrm{m}$. The

agreement of the ${ }^{3} \mathrm{He}$ and ${ }^{129} \mathrm{Xe}$ MRI-based maximal diffusion length scales (the diffusion lengths obtained for maximum diffusion coefficients of ${ }^{3} \mathrm{He}$ and ${ }^{129} \mathrm{Xe}$ in lungs) suggests the agreement of the mean diffusion lengths for chosen diffusion parameters. The proximity of the $\mathrm{Lm}_{\mathrm{D}}^{\mathrm{He}}$ and $\mathrm{Lm}_{\mathrm{D}}^{\mathrm{xe}}$ estimates was previously observed for a small group of COPD patients, where longer $\Delta_{\mathrm{Xe}}(8.5 \mathrm{~ms})$ was used for inhaled ${ }^{129} \mathrm{Xe} / \mathrm{N}_{2}$ gas mixture. ${ }^{39}$

Third, in the HV and AATD subgroups the additional emphysema biomarkers such as static-ventilation and $\mathrm{T}_{2}^{*}$ maps were obtained in a single breath-hold along with the ADC and morphometry maps. A consideration of the staticventilation based ventilation-defect-percent $(\mathrm{VDP})^{3}$ is out of the scope of this work, although, an example of the staticventilation image or short-TE, $b=0$ image is shown in Figure 1C. The image suggests that the achievable SNR level ( 60) should be sufficient for the VDP estimates calculation. However, we should note the slice thickness $(30 \mathrm{~mm})$ used in this work was larger than normally required for accurate
VDP calculations. ${ }^{40}$ The feasibility of the VDP calculation using short $\mathrm{TE} \mathrm{b}=0$ image has been recently demonstrated in an accelerated ${ }^{3} \mathrm{He}$ MRI morphometry study with a small number of the COPD subjects, where the slice thickness was $15 \mathrm{~mm} .{ }^{18} \mathrm{AF}=10$ (13 k-space lines out of 128 per image) can be used for accelerated ${ }^{129} \mathrm{Xe}$ MRI morphometry measurements to reduce the slice thickness down to $15 \mathrm{~mm}$ and keep the breath-hold time under the $16 \mathrm{~s}$. The feasibility of the AF = 10 approach has been recently demonstrated for a single young healthy subject. ${ }^{41}$ The expected SNR loss of the short $\mathrm{TE} b=0$ image because of the smaller slice volume can be compensated using the larger flip angle (less gradient steps in the y-direction) and more sensitive RF coils, specifically the multi-channel phased receive arrays. ${ }^{42}$

${ }^{129} \mathrm{Xe}$ MRI T ${ }_{2}^{*}$ values were previously estimated in volunteers on 3T Philips $(18 \pm 6 \mathrm{~ms})$ MRI systems ${ }^{20}$ Our mean $\mathrm{T}_{2}^{*}$ values obtained for HV were in the same range (12-16 ms), and this suggests that the apparent transverse relaxation time constant estimates we used were not inappropriate. We observed a linear relation between $\mathrm{Lm}_{\mathrm{D}}$ and $\mathrm{T}_{2}^{*}$ values (Figure 4), which suggests that alveolar destruction "improves" $\mathrm{B}_{0}$ field homogeneity. Therefore, relatively slow-moving xenon molecules do not experience a large scale $\mathrm{B}_{0}$ field inhomogeneity at $\mathrm{TE}=10 \mathrm{~ms}$ and the signal life-time is longer. This means that healthy lung regions likely had shorter $\mathrm{T}_{2}^{*}$ relaxation time.

The heterogeneity of the apparent transverse relaxation time constants of the hyperpolarized gas within the lung microstructure has been previously investigated in a number of studies. It has been shown that the magnetic susceptibility difference between gas and pulmonary tissue and the blood capillary system greatly contribute to the local magnetic field inhomogeneity. ${ }^{43}$ The referenced study suggests that the magnetic susceptibility effect should be larger for the lungs of young healthy subjects where the blood capillary system in the lung microstructure is normal and fully functional compared to the subjects with the moderate and sever emphysema where blood supply of the destroyed alveolus does not exist.

The signal void observed on the $\mathrm{b}=0$ long-TE image (Figure 1C, yellow arrow) corresponds to a short $\mathrm{T}_{2}^{*}(3-5 \mathrm{~ms})$ lung region. We have calculated the $b$-value corresponding to the readout gradient $\left(0.26 \mathrm{~s} / \mathrm{cm}^{2}\right)$ following the method of Ouriadov et $\mathrm{al}^{44}$ to check if the induced diffusion signal attenuation because of the imaging gradient contributes to the signal void. This b-value can cause only a 5\% signal decay for the case of freely diffusion xenon $\left(0.21 \mathrm{~cm}^{2} / \mathrm{s}\right)$. It is unlikely that a $5 \%$ diffusion decay was sufficient to significantly contribute to the signal void. The relationship between ${ }^{129} \mathrm{Xe}$ morphometry and apparent transverse relaxation can be potentially used for the longitudinal observation of the emphysema patients having non-ventilated lung regions as a result of the disease progression. Clearly, such regions cannot be visualized with the hyperpolarized gas MRI. However, the use of ${ }^{1} \mathrm{H}$ MRI T ${ }_{2}^{*}$ 
measurements can permit generation of diffusion estimates for such lung regions, assuming that there is also a correlation between $\mathrm{T}_{2}^{*}$ values obtained with hyperpolarized gas and proton MRI. Further investigations are required to determine the relationship between ${ }^{129} \mathrm{Xe}$ and ${ }^{1} \mathrm{H}$ MRI-based $\mathrm{T}_{2}^{*}$.

We acknowledge a number of limitations of this proofof-concept study and 1 of them was that the comparisons were not fully performed on a pixel-by-pixel basis for the data sets obtained using normal sampling, accelerate acquisitions, and multi-breath approach. Supporting Information Figure S1A and B demonstrate the relationships for ADC and $\mathrm{Lm}_{\mathrm{D}}$ obtained for all AATD subjects and all pixels. However, we believe that the plots are not very conclusive because of the lung volume mismatch and slice mismatch present between acquisitions. This is because of the variability inherent to multi-breath scanning but mostly because of the requirement to have the patients resting outside of the scanner between scans. The small difference in statistical plots visible in Supporting Information Figures S2 and S3 also indicates this. The slice mismatch can be seen in Figure 3, subject AATD-1. Therefore, we compared the global mean ADC and morphometry values obtained for these 3 cases because of the slice mismatch caused by breath-hold levels and patient-position changes between breath-holds. We think that the derived global mean ADC and morphometry do not influence our conclusions; a pixel-by-pixel comparison of the diffusion-weighted ${ }^{129} \mathrm{Xe}$ MRI data sets was retrospectively conducted in a small group of COPD patients as a validation step of the ${ }^{129} \mathrm{Xe}$ SEM-based morphometry with $\mathrm{AF}=7 .{ }^{18}$ Another limitation was the use of multi-breath data without co-registration to minimize a potential volume mismatch. Currently, we are in the process of optimizing a convexoptimization based deformable registration approach to address this issue. However, a convex optimization-based registration approach ${ }^{45}$ led to changes in background noise that meant that the SEM-based fitting could not be used. As a result, all ADC and morphometry values generated from the data sets corrected with the convex optimization-based registration approach were significantly underestimated. We used the diffusion-weighted multi-breath data in the previous studies and any significant influence on the ADC or morphometry estimates was not observed. ${ }^{15}$

\section{5 | CONCLUSION}

In this study, we did not statistically compare ADC and morphometry estimates acquired in the 2 subgroups because of their age difference and the small number of HV. As expected, the mean ADC and morphometry estimates calculated for the volunteers were smaller than the in AATD patients likely because of emphysema and age. ${ }^{46,47}$

In summary, single breath-hold multiple b-value whole lung accelerated $(\mathrm{AF}=7){ }^{129} \mathrm{Xe}$ MRI was demonstrated to be feasible in volunteers and patients. Further development of this method $(\mathrm{AF}=10)$ will potentially permit a single 16 -s breath-hold, whole lung ${ }^{129} \mathrm{Xe}$ MRI acquisition delivering static-ventilation, $\mathrm{T}_{2}^{*}, \mathrm{ADC}, \mathrm{Lm}_{\mathrm{D}}$, and $\mathrm{L}_{\mathrm{m}}$ maps with excellent spatial resolution. ${ }^{18}$

\section{ACKNOWLEDGMENTS}

G.P. gratefully acknowledges support from the Canadian Institutes of Health Research (CIHR) for research funding (operating grant MOP 106437 and the Canadian Respiratory Research Network) as well as the Natural Sciences and Engineering Research Council (Canada) Discovery and Accelerator awards. A.O. was funded in part by a grant from the Alpha-1 Foundation (USA). We thank J.F.P.J. Abascal for providing the MATLAB code for image reconstruction.

\section{ORCID}

Alexei Ouriadov (D) https://orcid.org/0000-0002-0920-2528

\section{REFERENCES}

1. Mugler JP 3rd, Altes TA. Hyperpolarized 129Xe MRI of the human lung. J Magn Reson Imaging. 2013;37:313-331.

2. Driehuys B, Martinez-Jimenez S, Cleveland ZI, et al. Chronic obstructive pulmonary disease: safety and tolerability of hyperpolarized 129Xe MR imaging in healthy volunteers and patients. Radiology. 2012;262:279-289.

3. Kirby M, Svenningsen S, Owrangi A, et al. Hyperpolarized $3 \mathrm{He}$ and 129Xe MR imaging in healthy volunteers and patients with chronic obstructive pulmonary disease. Radiology. 2012;265:600-610.

4. Kaushik SS, Cleveland ZI, Cofer GP, et al. Diffusion-weighted hyperpolarized 129Xe MRI in healthy volunteers and subjects with chronic obstructive pulmonary disease. Magn Reson Med. 2011;65:1154-1165.

5. Kirby M, Ouriadov A, Svenningsen S, et al. Hyperpolarized $3 \mathrm{He}$ and 129Xe magnetic resonance imaging apparent diffusion coefficients: physiological relevance in older never- and ex-smokers. Physiol Rep. 2014;2:e12068.

6. Bhandari A, Bhandari V. Pathogenesis, pathology and pathophysiology of pulmonary sequelae of bronchopulmonary dysplasia in premature infants. Front Biosci. 2003;8:e370-380.

7. Bals R. Alpha-1-antitrypsin deficiency. Best Pract Res Clin Gastroenterol. 2010;24:629-633.

8. Sheikh K, Bhalla A, Ouriadov A, et al. Pulmonary magnetic resonance imaging biomarkers of lung structure and function in adult survivors of bronchopulmonary dysplasia with COPD. Cogent Med. 2017;4:1282033.

9. Ouriadov A, Lessard E, Sheikh K, Parraga G, Canadian Respiratory Research N. Pulmonary MRI morphometry modeling of airspace enlargement in chronic obstructive pulmonary disease and alpha-1 antitrypsin deficiency. Magn Reson Med. 2018;79:439-448.

10. Thomen RP, Quirk JD, Roach D, et al. Direct comparison of (129) Xe diffusion measurements with quantitative histology in human lungs. Magn Reson Med. 2017;77:265-272.

11. Stewart NJ, Norquay G, Marshall H, Wild JM. Feasibility of high quality lung MRI with naturally-abundant hyperpolarised 129Xe. Eur Respir J. 2014;44:1729.

12. Kaushik SS, Robertson SH, Freeman MS, et al. Single-breath clinical imaging of hyperpolarized (129)Xe in the airspaces, barrier, 
and red blood cells using an interleaved 3D radial 1-point Dixon acquisition. Magn Reson Med. 2016;75:1434-1443.

13. Zhang H, Xie J, Xiao S, et al. Lung morphometry using hyperpolarized (129) Xe multi-b diffusion MRI with compressed sensing in healthy subjects and patients with COPD. Med Phys. 2018;45:3097-3108.

14. Chang YV, Quirk JD, Yablonskiy DA. In vivo lung morphometry with accelerated hyperpolarized 3He diffusion MRI: a preliminary study. Magn Reson Med. 2015;73:1609-1614.

15. Ouriadov A, Farag A, Kirby M, McCormack DG, Parraga G, Santyr GE. Lung morphometry using hyperpolarized (129) Xe apparent diffusion coefficient anisotropy in chronic obstructive pulmonary disease. Magn Reson Med. 2013;70:1699-1706.

16. Chan HF, Stewart NJ, Parra-Robles J, Collier GJ, Wild JM. Whole lung morphometry with 3D multiple b-value hyperpolarized gas MRI and compressed sensing. Magn Reson Med. 2017; 77:1916-1925.

17. Abascal JFPJ, Desco M, Parra-Robles J. Incorporation of prior knowledge of the signal behavior into the reconstruction to accelerate the acquisition of MR diffusion data. arXiv e-prints arXiv. 2017:1702.02743.

18. Westcott A, Guo F, Parraga G, Ouriadov A. Rapid single-breath hyperpolarized noble gas MRI-based biomarkers of airspace enlargement. J Magn Reson Imaging. 2019;49:1713-1722.

19. Parra-Robles J, Dominguez Viqueira W, Xu X, Ouriadov A, Santyr GE. Theoretical prediction and experimental measurement of the field dependence of the apparent transverse relaxation of hyperpolarized noble gases in lungs. J Magn Reson. 2008;192:85-91.

20. Xu X, Norquay G, Parnell SR, et al. Hyperpolarized 129Xe gas lung MRI-SNR and T2* comparisons at $1.5 \mathrm{~T}$ and $3 \mathrm{~T}$. Magn Reson Med. 2012;68:1900-1904.

21. Komlosi P, Altes TA, Qing K, et al. Signal-to-noise ratio, T2, and $\mathrm{T} 2 *$ for hyperpolarized helium-3 MRI of the human lung at three magnetic field strengths. Magn Reson Med. 2017;78:1458-1463.

22. Miller MR, Hankinson J, Brusasco V, et al. Standardisation of spirometry. Eur Respir J. 2005;26:319-338.

23. Ouriadov A, Farag A, Kirby M, McCormack DG, Parraga G, Santyr GE. Pulmonary hyperpolarized (129) Xe morphometry for mapping xenon gas concentrations and alveolar oxygen partial pressure: proof-of-concept demonstration in healthy and COPD subjects. Magn Reson Med. 2015;74:1726-1732.

24. Hersman FW, Ruset IC, Ketel S, et al. Large production system for hyperpolarized 129Xe for human lung imaging studies. Acad Radiol. 2008;15:683-692.

25. Fichele S, Woodhouse N, Swift AJ, et al. MRI of helium-3 gas in healthy lungs: posture related variations of alveolar size. J Magn Reson Imaging. 2004;20:331-335.

26. Tsai LL, Mair RW, Li CH, Rosen MS, Patz S, Walsworth RL. Posture-dependent human $3 \mathrm{He}$ lung imaging in an open-access MRI system: initial results. Acad Radiol. 2008;15:728-739.

27. Parra-Robles J, Marshall H, Hartley RA, Brightling CE, Wild JM. Quantification of lung microstructure in asthma using a $3 \mathrm{He}$ fractional diffusion approach. In Proceedings of the 22nd Annual Meeting of ISMRM, Milan, Italy, 2014. Abstract 3529.

28. Berberan-Santos MN, Bodunov EN, Valeur B. Mathematical functions for the analysis of luminescence decays with underlying distributions 1. Kohlrausch decay function (stretched exponential). Chem Phys. 2005;315:171-182.

29. Ouriadov A, Lessard E, Guo F, et al. Accelerated diffusion-weighted 129Xe MRI morphometry of emphysema in COPD and alpha-1 antitrypsin deficiency patients. In Proceedings of the 25th Annual Meeting of ISMRM, Honolulu, HI, 2017. Abstract 1763.

30. Goldstein T, Osher S. The split Bregman method for L1-regularized problems. SIAM J Imaging Sci. 2009;2:323-343.

31. Sukstanskii AL, Yablonskiy DA. In vivo lung morphometry with hyperpolarized $3 \mathrm{He}$ diffusion MRI: theoretical background. J Magn Reson. 2008;190:200-210.

32. Yablonskiy DA, Sukstanskii AL. Chapter 12 - Lung morphometry with HP gas diffusion MRI: from theoretical models to experimental measurements A2 - Albert, Mitchell S. In: Hane FT, ed. Hyperpolarized and inert gas MRI. Boston: Academic Press; 2017:183-209.

33. Sukstanskii AL, Yablonskiy DA. Lung morphometry with hyperpolarized 129Xe: theoretical background. Magn Reson Med. 2012;67:856-866.

34. van Beek EJR, Dahmen AM, Stavngaard T, et al. Hyperpolarised 3He MRI versus HRCT in COPD and normal volunteers: PHIL trial. Euro Respir J. 2009;34:1311-1321.

35. Diaz S, Casselbrant I, Piitulainen E, et al. Progression of emphysema in a 12-month hyperpolarized 3He-MRI study: lacunarity analysis provided a more sensitive measure than standard ADC analysis. Acad Radiol. 2009;16:700-707.

36. Diaz S, Casselbrant I, Piitulainen E, et al. Hyperpolarized 3He apparent diffusion coefficient MRI of the lung: reproducibility and volume dependency in healthy volunteers and patients with emphysema. J Magn Reson Imaging. 2008;27:763-770.

37. Stavngaard T, Sogaard LV, Batz M, Schreiber LM, Dirksen A. Progression of emphysema evaluated by MRI using hyperpolarized (3) $\mathrm{He}(\mathrm{HP}$ (3)He) measurements in patients with alpha-1-antitryp$\sin$ (A1AT) deficiency compared with CT and lung function tests. Acta Radiol. 2009;50:1019-1026.

38. Ruppert K, Quirk JD, Mugler JPI, et al. Lung morphometry using hyperpolarized xenon-129: preliminary experience. In Proceedings of the 20th Annual Meeting of ISMRM, Melbourne, Australia, 2012. Abstract 1352.

39. Chan HF, Collier GJ, Weatherley ND, Wild JM. Comparison of in vivo lung morphometry models from 3D multiple b-value (3) $\mathrm{He}$ and (129) Xe diffusion-weighted MRI. Magn Reson Med. 2019;81:2959-2971.

40. Kirby M, Heydarian M, Svenningsen S, et al. Hyperpolarized $3 \mathrm{He}$ magnetic resonance functional imaging semiautomated segmentation. Acad Radiol. 2012;19:141-152.

41. Ouriadov A, Westcott A, McCormack DG, Parraga G. Feasibility of 129Xe MRI morphometry measurements with acceleration factors of 7 and 10. In Proceedings of the 27th Annual Meeting of ISMRM, Montreal, Canada, 2019. Abstract 3518.

42. Zanette B, Santyr G. Accelerated interleaved spiral-IDEAL imaging of hyperpolarized (129) Xe for parametric gas exchange mapping in humans. Magn Reson Med. 2019;82:1113-1119.

43. Vignaud A, Maître X, Guillot G, et al. Magnetic susceptibility matching at the air-tissue interface in rat lung by using a superparamagnetic intravascular contrast agent: influence on transverse relaxation time of hyperpolarized helium-3. Magn Reson Med. 2005;54:28-33.

44. Ouriadov AV, Santyr GE. High spatial resolution hyperpolarized (3) He MRI of the rodent lung using a single breath X-centric gradient-recalled echo approach. Magn Reson Med. 2017;78:2334-2341.

45. Guo F, Svenningsen S, Kirby M, et al. Thoracic CT-MRI coregistration for regional pulmonary structure-function measurements of obstructive lung disease. Med Phys. 2017;44:1718-1733. 
46. Fain SB, Altes TA, Panth SR, et al. Detection of age-dependent changes in healthy adult lungs with diffusion-weighted 3He MRI. Acad Radiol. 2005;12:1385-1393.

47. Quirk JD, Sukstanskii AL, Woods JC, et al. Experimental evidence of age-related adaptive changes in human acinar airways. $J$ Appl Physiol (1985). 2016;120:159-165.

\section{SUPPORTING INFORMATION}

Additional supporting information may be found online in the Supporting Information section.

FIGURE S1 Relationships for $\mathrm{ADC}$ and $\mathrm{Lm}_{\mathrm{D}}$ obtained for all AATD subjects and all pixels. (A) $\mathrm{ADC}^{\mathrm{A}}$ versus $\mathrm{ADC}$ relationship $(\mathrm{R}=0.65, P<0.0001)$ and $(\mathrm{B}) \mathrm{Lm}_{\mathrm{D}}{ }^{\mathrm{A}} \mathrm{vs} \mathrm{Lm}_{\mathrm{D}}$ relationship $(\mathrm{R}=0.65, P<0.0001) .{ }^{\mathrm{A}}$ indicates undersampling with the acceleration factor of 7

FIGURE S2 statistical plots obtained for AATD-1 subject (Figure 3) for (A) ADC $\left(\mathrm{ADC}^{\mathrm{A}}\right)$ maps and (B) $\mathrm{Lm}_{\mathrm{D}}\left(\mathrm{Lm}_{\mathrm{D}}{ }^{\mathrm{A}}\right)$ maps. ${ }^{\mathrm{A}}$ indicates undersampling with the acceleration factor of 7

FIGURE S3 statistical plots obtained for AATD-2 subject (Figure 3) for (A) $\mathrm{ADC}\left(\mathrm{ADC}{ }^{\mathrm{A}}\right)$ maps and (B) $\mathrm{Lm}_{\mathrm{D}}\left(\mathrm{Lm}_{\mathrm{D}}{ }^{\mathrm{A}}\right)$ maps. ${ }^{\mathrm{A}}$ indicates undersampling with the acceleration factor of 7

FIGURE S4 Histograms obtained for $2 \mathrm{HV}$ and 2 AATD subjects for ADC maps presented in Figures 2 and 3. (A)
ADC distribution obtained for HV-1 and AATD-1. (B) ADC distribution obtained for HV-2 and AATD-2. Only fully sampled data were used to generated the ADC maps to avoid any errors assisted with the compressed sampling

TABLE S1 Pulmonary function measurements, demographic, and ${ }^{129} \mathrm{Xe}$ MRI Parenchyma Measurements in AATD. BMI, body mass index; $\mathrm{FEV}_{1}$, forced expiratory volume in $1 \mathrm{~s} ; \%_{\text {pred }}$, percent-predicted; FVC, forced vital capacity; $\mathrm{RV}$, residual-volume; $\mathrm{DL}_{\mathrm{CO}}$, diffusing capacity of carbon monoxide; AATD, alpha-one antitrypsin deficiency; AF, acceleration factor; $\mathrm{T}_{2}^{*}$, apparent transverse relaxation time constant; ADC, apparent diffusion coefficient; $\mathrm{Lm}_{\mathrm{D}}$, mean diffusion length; $\mathrm{L}_{\mathrm{m}}$, mean linear intercept estimate. The $\mathrm{T}_{2}^{*}$ estimates (fully sampled data) for subjects 1 and 2 were not calculated due the image artefacts (scanner's receiver saturation) appeared on the short $\mathrm{TE} b=0$ image

How to cite this article: Ouriadov A, Guo F, McCormack DG, Parraga G. Accelerated ${ }^{129}$ Xe MRI morphometry of terminal airspace enlargement: Feasibility in volunteers and those with alpha-1 antitrypsin deficiency. Magn Reson Med. 2019;00: 1-11. https://doi.org/10.1002/mrm.28091 